Groups Geom. Dyn. 5 (2011), 673-681

DOI $10.4171 / \mathrm{GGD} / 143$
Groups, Geometry, and Dynamics

(C) European Mathematical Society

\title{
How to read the length of a braid from its curve diagram
}

\author{
Bert Wiest
}

\begin{abstract}
We prove that the Garside length of a braid is equal to a winding-number type invariant of the curve diagram of the braid.
\end{abstract}

Mathematics Subject Classification (2010). 20F36, 20 F10.

Keywords. Braid group, Garside group, curve diagram.

\section{Introduction}

Is it possible to read the length of a braid $\beta$ from the curve diagram of $\beta$ ? The words used in this question admit different interpretations, but in this paper we shall show that, if the word "length" is interpreted in the sense of Garside, then the answer is affirmative: the Garside length of a braid is equal to a winding-number type invariant of the curve diagram which can be read from the diagram by a simple-minded procedure.

The easiest answer one might have hoped for is that the length of a braid is proportional to the number of intersections of the curve diagram of the braid with the real line. This answer, however, is false - see for instance [12] and [4]. In these papers a relation was established between a certain distorted word length and the above-mentioned intersection number, which is in turn related to the distance in Teichmüller space between a base point and its image under the braid action.

Let us recall some basic definitions and establish some notation. We recall that Garside introduced in [8] a certain set of generators for the braid group, called the Garside generators (or "divisors of $\Delta$ " or "positive permutation braids"). The length of an element of the braid group with respect to this generating set is called its Garside length. Still according to Garside, every braid $\beta$ can be written in a canonical way as a product of Garside generators and their inverses (see e.g. [5], [2], [9]). From this canonical form one can read off two integer numbers $\inf (\beta)$ and $\sup (\beta)$, which are the maximal and minimal integers, respectively, satisfying

$$
\Delta^{\inf (\beta)} \preccurlyeq \beta \preccurlyeq \Delta^{\sup (\beta)} .
$$


(Here the symbol $\preccurlyeq$ denotes the subword partial ordering: $\beta_{1} \preccurlyeq \beta_{2}$ means that there exists a product $w$ of Garside generators such that $\beta_{1} \cdot w=\beta_{2}$. Moreover, $\Delta$ denotes Garside's half twist braid $\sigma_{1} \cdot\left(\sigma_{2} \sigma_{1}\right) \ldots\left(\sigma_{n-1} \ldots \sigma_{1}\right)$.) Then one can show that the Garside length of $\beta$ equals $\max (\sup (\beta), 0)-\min (\inf (\beta), 0)$.

We denote $D_{n}$ the $n$ times punctured disk, i.e., the disk $D^{2}=\{c \in \mathbb{C}|| c \mid \leqslant 1\}$, equipped with $n$ punctures which are regularly spaced in the interior of the interval $[-1,1]$. We define $E$ to be the diagram in $D_{n}$ consisting of the real (horizontal) line segment between the leftmost and the rightmost puncture. We define $\bar{E}$ to be the diagram consisting of the real line segment between the point -1 (the leftmost point of $\partial D^{2}$ ) and the rightmost puncture. A curve diagram of a braid $\beta$ is the image of $\bar{E}$ under a diffeomorphism of $D_{n}$ representing the braid $\beta$ (cf. [6]). Throughout this paper, braids act on the right. We shall call a curve diagram reduced if it has the minimal possible number of intersections with the horizontal line, and also the minimal possible number of vertical tangencies in its diffeotopy class, and if none of the punctures lies in a point of vertical tangency.

\section{Winding number labellings and the main result}

For a braid $\beta$ we are going to denote $\bar{D}_{\beta}$ the curve diagram of $\beta$, and $D_{\beta}$ the image of $E$ under $\beta$ - so $D_{\beta}$ is obtained from $\bar{D}_{\beta}$ simply by removing one arc. We shall label each segment of $\bar{D}_{\beta}$ between two subsequent vertical tangencies by an integer number, in the following way: the first segment (which starts on $\partial D^{2}$ ) is labelled 0 , and if the label of the $i$ th segment is $k$, and if the transition from the $i$ th to the $(i+1)$ st segment is via a right curve, then the $(i+1)$ st segment is labelled $k+1$. If, on the other hand, the transition is via a left curve, then the label of the $(i+1)$ st segment is $k-1$. See Figure 1 for an example of this labelling. We shall call this labelling the winding number labelling of the curve diagram.

A more rigorous definition of this labelling is as follows. If $\alpha: I \rightarrow D^{2}$ is a smooth parametrization of the curve diagram $\bar{D}_{\beta}$, defined on the unit interval $I=[0,1]$, and such that $\alpha(0)=-1$, then we define the tangent direction function $T_{\alpha}: I \rightarrow \mathbb{R} / 2 \mathbb{Z}$ as the angle of the tangent direction of $\alpha$ against the horizontal, divided by $-\pi$. In particular, if the arc goes straight to the right in $\alpha(t)$, then $T_{\alpha}(t)=0+2 \mathbb{Z}$. If it goes straight down, then $T_{\alpha}(t)=\frac{1}{2}+2 \mathbb{Z}$; and if it goes to the left, then $T_{\alpha}(t)=1+2 \mathbb{Z}$. Now we have a unique lifting of the function $T_{\alpha}$ to a function $\widetilde{T}_{\alpha}: I \rightarrow \mathbb{R}$ with $\widetilde{T}_{\alpha}(0)=0$. Finally, if $r: \mathbb{R} \rightarrow \mathbb{Z}$ denotes the rounding function, which sends every real number to the nearest integer (rounding down $n+\frac{1}{2}$ ), then we define the function

$$
\tau_{\alpha}: I \rightarrow \mathbb{R}, \quad t \mapsto r \circ \widetilde{T}_{\alpha}(t),
$$

which one might call the rounded lifted tangent direction function.

Now the winding number labelling can be redefined as follows: a point $x$ of $D_{\beta}$ with non-vertical tangent direction is labelled by the integer $\tau_{\alpha}(t)$, where $t$ in $I$ is 
such that $\alpha(t)=x$.

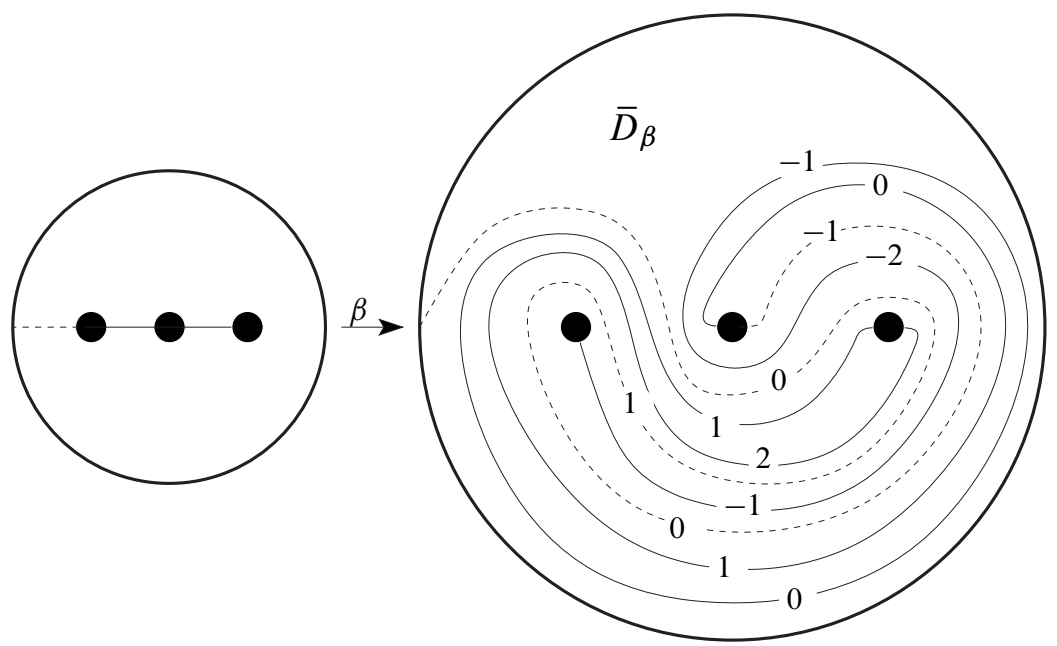

Figure 1. The curve diagram (with the first line drawn dashed) of the braid $\beta=\left(\sigma_{1} \sigma_{2}^{-1}\right)^{2}$. The labels of the solid arcs vary between -2 and 2. The Garside normal form of $\beta$ is $\sigma_{2}^{-1} \sigma_{1}^{-1}$. $\sigma_{1}^{-1} \cdot \sigma_{2} \cdot \sigma_{2} \sigma_{1}$, so its infimum is -2 and its supremum is 2 . Also note that there is only one arc carrying the maximal label " 2 " and only one arc with the minimal label " -2 ".

We shall denote the largest and smallest labels occurring in $D_{\beta}$ by $\operatorname{LL}(\beta)$ and $\operatorname{SL}(\beta)$. Notice that we are talking about the restricted diagram $D_{\beta}$, not the full diagram $\bar{D}_{\beta}$. Nevertheless, in order to actually calculate the labels of the restricted diagram, one has to start by calculating the labels of the first arc (the one that starts at -1 on $\left.\partial D^{2}\right)$.

Theorem 2.1. For any braid $\beta$, the largest label occurring in the diagram $D_{\beta}$ is equal to $\sup (\beta)$ and the smallest label occurring is equal to $\inf (\beta)$. In particular, the Garside length of the braid $\beta$ is $\max (\operatorname{LL}(\beta), 0)-\min (\operatorname{SL}(\beta), 0)$.

Proof of Theorem 2.1. The proof comes down to the following lemma:

Lemma 2.2. If $\beta$ is a positive braid, then the largest label occurring in the diagram $D_{\beta}$ is equal to the Garside length of $\beta$.

Let us first see why Lemma 2.2 implies Theorem 2.1: let us assume for the moment that Lemma 2.2 is true, and try to deduce Theorem 2.1. The crucial observation is that multiplying $\beta$ by $\Delta^{k}$ increases all four numbers (the sup, the inf, the maximal 
label of $D_{\beta}$ and the minimal label of $D_{\beta}$ ) by $k$. We obtain

$$
\begin{aligned}
\operatorname{LL}(\beta) & =\operatorname{LL}\left(\beta \Delta^{-\inf (\beta)}\right)+\inf (\beta) \\
& \stackrel{*}{=} \text { length }_{\text {Gars }}\left(\beta \Delta^{-\inf (\beta)}\right)+\inf (\beta) \\
& =\sup \left(\beta \Delta^{-\inf (\beta)}\right)+\inf (\beta)=\sup (\beta),
\end{aligned}
$$

where $\stackrel{*}{=}$ denotes that the equality follows from Lemma 2.2 . Now we make the following

Claim: If $\beta$ is a negative braid, then the smallest label occurring in $D_{\beta}$, multiplied by -1 , is equal to the Garside length of $\beta$.

In order to prove this claim, we consider the image $\bar{\beta}$ of $\beta$ under the homomorphism which replaces each crossing $\sigma_{i}^{ \pm 1}$ by its negative crossing $\sigma_{i}^{\mp 1}$. Its curve diagram $D_{\bar{\beta}}$ is just the mirror image, with respect to the horizontal line, of $D_{\beta}$. We observe that

$$
\inf (\beta)=-\sup (\bar{\beta}), \quad \sup (\beta)=-\inf (\bar{\beta}), \operatorname{LL}(\beta)=-\operatorname{SL}(\bar{\beta}), \operatorname{SL}(\beta)=-\operatorname{LL}(\bar{\beta}) .
$$

The claim now follows from Lemma 2.2.

Now we have for an arbitrary braid $\beta$ that

$$
\begin{aligned}
\operatorname{SL}(\beta) & =\operatorname{SL}\left(\beta \Delta^{-\sup (\beta)}\right)+\sup (\beta) \\
& \stackrel{*}{=}-\text { length }_{\operatorname{Gars}}\left(\beta \Delta^{-\sup (\beta)}\right)+\sup (\beta) \\
& =\inf \left(\beta \Delta^{-\sup (\beta)}\right)+\sup (\beta)=\inf (\beta),
\end{aligned}
$$

where $\stackrel{*}{=}$ denotes that the equality follows from the Claim. This completes the proof of Theorem 2.1, assuming Lemma 2.2.

Proof of Lemma 2.2. First we shall prove that for a positive braid $\beta$ we have $\operatorname{LL}(\beta) \leqslant$ length $_{\text {Gars }}(\beta)$. By induction, this is equivalent to proving the following: if $\beta$ is a positive braid and $\beta_{+}$is a divisor of $\Delta$, then $\operatorname{LL}\left(\beta \cdot \beta_{+}\right) \leqslant \operatorname{LL}(\beta)+1$.

The action on the disk of any braid $\beta_{+}$which is a divisor of $\Delta$ can be realized by the following dance of the punctures. Initially the punctures are lined up on the real line. As a first step, perform a clockwise rotation so that the punctures are lined up on the imaginary axis. As a second step, move each of the punctures horizontally, until no more puncture lies precisely above any other one. In a third step, by vertical movements, bring all the punctures back to the horizontal line.

Let us suppose that $\alpha: I \rightarrow D^{2}$ is a parametrization of the curve diagram $\bar{D}_{\beta}$, and let us look at a segment $I^{\prime} \subseteq I$ parametrizing a segment between two successive points of vertical tangency. The function $\left.\tau_{\alpha}\right|_{I^{\prime}}$ is constant, we shall denote its value by $k$; this means that on the interval $I^{\prime}$, the function $\widetilde{T}_{\alpha}$ takes values in the interval ]$\left.k-\frac{1}{2}, k+\frac{1}{2}\right]$. Now, after the first step of the puncture dance (the rotation), we have a deformed curve diagram parametrized by a function $\alpha^{\prime}: I \rightarrow D^{2}$, and we 
observe that on the interval $I^{\prime}$, the function $\widetilde{T}_{\alpha^{\prime}}$ takes values in the interval $\left.] k, k+1\right]$. The horizontal movement of the punctures deforms the arc $\alpha^{\prime}$ into an arc $\alpha^{\prime \prime}$, but during this deformation no horizontal tangencies are created or destroyed; therefore the values of $\left.\widetilde{T}_{\alpha^{\prime \prime}}\right|_{I^{\prime}}$ still lie in the interval $\left.] k, k+1\right]$. The third step (squashing the punctures back to the real line) changes tangent directions by at most a quarter turn, so the new function $\widetilde{T}_{\alpha^{\prime \prime \prime}}$ takes values in the interval $\left.] k-\frac{1}{2}, k+\frac{3}{2}\right]$ on $I^{\prime}$. Thus on the interval $I^{\prime}$, we have $\tau_{\alpha^{\prime \prime \prime}} \in\{k, k+1\}$, so the labels have increased by at most one under the action of $\beta_{+}$. This completes the proof that $\operatorname{LL}(\beta) \leqslant$ length $_{\operatorname{Gars}}(\beta)$.

In order to prove the converse inequality, we shall prove that for any braid $\beta$ with $\operatorname{SL}(\beta) \geqslant 0$, there is a braid $\beta_{-}$which is the inverse of a simple braid such that $\operatorname{LL}\left(\beta \cdot \beta_{-}\right)=\operatorname{LL}(\beta)-1$ and still $\operatorname{SL}\left(\beta \cdot \beta_{-}\right) \geqslant 0$. Intuitively, every braid can be "relaxed" into another one with less high twisting.

Our construction of such a braid $\beta_{-}$will again be in the form of a dance of the punctures of $D_{\beta}$, where in a first step each puncture performs a vertical movement until no two punctures lie at the same height, in a second step the punctures are squashed onto the imaginary axis by horizontal movements, and in a third step the punctures are brought back to the horizontal axis by a $90^{\circ}$ counterclockwise rotation of the vertical axis.

The only step that needs to be defined in a more detailed manner is the first one. In order to do so, we first classify the kinds of segments which one can see between successive points with vertical tangency in the diagram $D_{\beta}$. Firstly, there are those segments which start in a right turn and end in a left turn (see Figure 2 (a)); these correspond to local maxima of the function $\tau_{\alpha}$. Secondly, there are those segments that start in a left and end in a right turn (see Figure 2 (b)); these correspond to local minima of the function $\tau_{\alpha}$. Thirdly, there are those that start and end in a right turn, and, fourthly, those that start and end in a left turn (see Figure 2 (c) and (d)). The key to the construction of $\beta_{-}$is the following lemma, which is also illustrated in Figure 2.

Sublemma 2.3. There exists a diffeotopy of the disk which moves every point only vertically up or down and which deforms the diagram $D_{\beta}$ into a diagram $D^{\prime}$ such that:

(1) Arcs of the first and second type in $D_{\beta}$ give rise to arcs in $D^{\prime}$ which do not have any horizontal tangencies.

(2) Arcs of the third type in $D_{\beta}$ give rise to arcs in $D^{\prime}$ which have precisely one maximum and no minimum in the vertical direction (and hence exactly one horizontal tangency).

(3) Arcs of the fourth type in $D_{\beta}$ give rise to arcs in $D^{\prime}$ which have precisely one minimum and no maximum in the vertical direction.

Proof of Sublemma 2.3. We define a relation on the set of punctures in the diagram $D_{\beta}$ by saying that a puncture $p_{1}$ is below a puncture $p_{2}$ if there is some segment of 
(a)

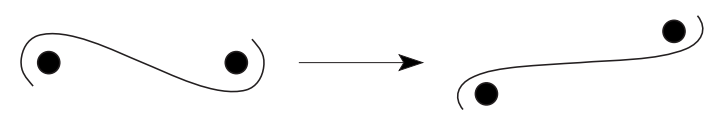

(b)

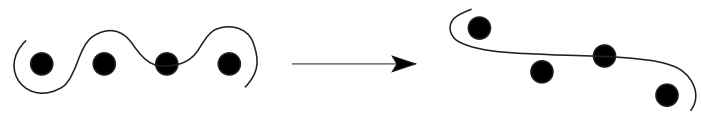

(c)

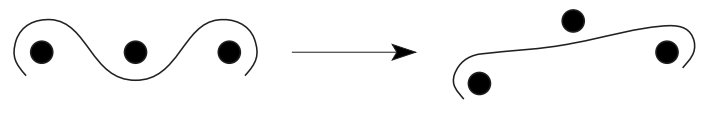

(d)

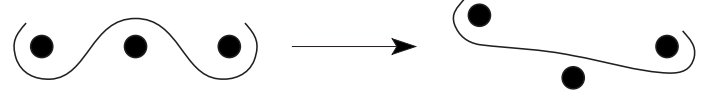

Figure 2. Four types of segments of curve between points of vertical tangency, and how to deform them by vertical movements of the punctures.

$D_{\beta}$ which contains no vertical tangencies and such that $p_{1}$ lies below the segment, or possibly on it, and such that $p_{2}$ lies above, or possibly on, the segment - but at most one of the two punctures is supposed to lie on the segment. We observe that this relation is a partial order. Let us choose any extension of this partial order to a total order. Now the desired diffeotopy can be obtained by sliding the punctures of $D_{\beta}$ up or down so that their vertical order corresponds to the total order just defined.

This completes our description of the first step of the puncture dance, and hence the definition of the braid $\beta_{-}$.

Now we have to prove that the action of $\beta_{-}$simplifies the curve diagram as claimed. Let us look at an arc of $D_{\beta}$ between two successive vertical tangencies which carries the label $\operatorname{LL}(\beta)$, i.e., the largest label that occurs. Such an arc is of the first type, in the above classification. Let us suppose that $\alpha: I \rightarrow D^{2}$ is a parametrization of the curve diagram $D_{\beta}$, and that $I^{\prime}$ is a subinterval of $I$ such that $\left.\alpha\right|_{I^{\prime}}$ parametrizes the arc under consideration. On the interval $I^{\prime}$, the function $T_{\alpha}$ takes values in the range ] $\left.\operatorname{LL}(\beta)-\frac{1}{2}, \operatorname{LL}(\beta)+\frac{1}{2}\right]$. The first step of our particle dance, however, deforms $\alpha$ into a parametrized arc $\alpha^{\prime}$ such that $T_{\alpha^{\prime}}$ only takes values in the range ] $\operatorname{LL}(\beta)-\frac{1}{2}, \operatorname{LL}(\beta)[$.

Now the second step deforms the arc $\alpha^{\prime}$ into an arc $\alpha^{\prime \prime}$. Since this step only moves points horizontally, it never creates or destroys a point of horizontal tangency in the diagram, so on the interval $I^{\prime}$ the function $T_{\alpha^{\prime \prime}}$ only takes values in the range ] $\operatorname{LL}(\beta)-1, \operatorname{LL}(\beta)\left[\right.$. Finally, the third step acts as a $90^{\circ}$ counterclockwise rotation on the arc, so the values of $T_{\alpha^{\prime \prime \prime}}$ lie in the interval ] $\operatorname{LL}(\beta)-\frac{3}{2}, \operatorname{LL}(\beta)-\frac{1}{2}[$. Therefore the segment parametrized by $\left.\alpha^{\prime \prime \prime}\right|_{I^{\prime}}$ is part of a segment in the curve diagram of $D_{\beta \beta_{+}}$ which is labelled $\operatorname{LL}(\beta)-1$.

Next we claim that an arc of $D_{\beta}$ which is labelled by $\operatorname{SL}(\beta)$, i.e., the minimal possible label, gives rise in $D_{\beta \beta_{+}}$to part of an arc which is still labelled $\operatorname{SL}(\beta)$, and 
in particular still positive. This proof is similar to the argument just presented, and it is left to the reader.

\section{Maximally labeled arcs are rare}

There is one additional observation to be made about our labellings of curve diagrams. Not only do the extremal labels determine the Garside length of the braid, but moreover these extremal labels occur only very rarely in the diagram: there is a bound on their number which is linear in the number of punctures $n$ and independent of $\beta$. More precisely:

Proposition 3.1. Suppose that $\beta$ is a braid with $n$ strands. Then in the winding number labelling of the curve diagram $\bar{D}_{\beta}$ there are at most $n-1$ arcs labelled $\operatorname{LL}(\beta)$, and there are at most $n-1$ arcs labelled $\operatorname{SL}(\beta)$.

Proof. We shall prove that there are at most $n-1$ arcs labelled $\operatorname{LL}(\beta)$ (the proof in the case of $\operatorname{SL}(\beta)$ is similar), and we shall do so by proving the following result:

Proposition 3.1'. No two of the arcs labelled $\operatorname{LL}(\beta)$ have their right extremities between the same pairs of punctures. The same holds for the left extremities.

Let us assume, for a contradiction, that two such arcs exist. Figure 3 illustrates what this means, in the particular case $\operatorname{LL}(\beta)=7$. There are different possibilities, depending on whether or not the rightmost puncture lies on one of the arcs.

(17)

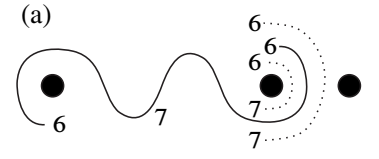

(1)

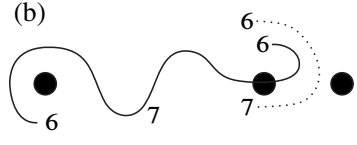

(c)

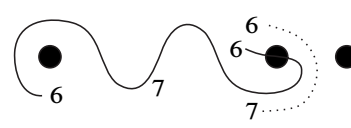

Figure 3. A schematic picture of a curve diagram with $\operatorname{LL}(\beta)=7$. Neither of the dotted lines can occur, with the required labels, in the prolongation of the solid line.

The proof is completed by checking all possible isotopy classes in $D^{2} \backslash$ (solid arc) of embedded arcs connecting one endpoint of the solid arc of Figure 3 to one of the endpoints of one of the dotted arcs. One observes that the labellings never match up.

\section{Outlook}

This paper was motivated by the question "What do quasi-geodesics in braid groups really look like?". More precisely, it is an experimental observation that any reasonable way of untangling the curve diagram of a braid $\beta$ yields a quasi-geodesic 
representative of $\beta$ [13]. However, the question what precisely is "reasonable" has turned out to be very difficult.

An essential portion of the answer seems to be provided by the insight of Masur and Minsky [11] that for any braid (or mapping class) there are only finitely many subsurfaces whose interior is tangled by the action of the braid, and by the MasurMinsky-Rafi distance formula [12]. It is, however, not clear how these results can be used in practice to prove, for instance, that the Bressaud normal form $[1,3]$ or the transmission-relaxation normal form [4] are quasi-geodesic, or that all braids have $\sigma$-consistent representatives of linearly bounded length [7]. It would be very useful to have a more practical, or algorithmic, version of these ideas.

Let us denote $\Delta_{i, j}$ the braid in which strands number $i, i+1, \ldots, j$ perform a half twist. Let us define the $\tau$-length of a Garside-generator in the following way: it is its length when written as a word in the generators $\Delta_{i, j}$ (equivalently, it is the number of half Dehn twists along round curves in $D_{n}$ needed to express it). Now, given a braid $\beta$, put it in right Garside normal form, and add up the lengths of its factors. We shall call the result the $\tau$-length of $\beta$.

For a subdisk $D \subseteq D_{n}$ which is round (contains punctures number $i, i+1, \ldots, j$ for some $1 \leqslant i<j \leqslant n$ ), and a braid $\beta$, we define the tangledness of the curve diagram of $\beta$ in $D$ in the following way. The intersection of the curve diagram of $\beta$ with $D$ consists of a (possibly very large) number of arcs, which inherit a labelling from the winding number labelling of the full diagram. Suppose that you shift the labelling of each arc inside the subdisk so that the two extremities of each arc

- either both lie in a segment labelled 0 , or

- one lies in a segment labelled 0 and the other lies in a segment labelled 1.

Now for each arc take

(the largest label - the smallest label) or (the largest label $-1-$ the smallest label)

according to the type of the arc. Take the maximum of these quantities over all arcs. That is the tangledness.

Conjecture 4.1. Suppose that in the curve diagram of a braid $\beta$ there is a round disk whose interior curve diagram has strictly positive tangledness. Suppose that $\beta_{+}$is a Garside generator or the inverse of a Garside generator, that it moves only strands inside the round disk, and that its action reduces the tangledness of the diagram inside the round disk. Then $\beta \beta_{+}$has smaller $\tau$-length than $\beta$.

Question 4.2. Another question also arises from Proposition 3.1'. For a pseudoAnosov braid $\beta$ we can look at the maximally and minimally labelled arcs in the curve diagrams of high powers of $\beta$, and, by passing to the limit, in a train track or in the stable foliation of $\beta$. The obvious question is: what do the positions of these arcs tell us? Could they, for instance, be helpful for solving the conjugacy problem? 
Question 4.3. Is there an analogue of the main result (Theorem 2.1) for $\operatorname{Out}\left(F_{n}\right)$, or at least a substantial subgroup of $\operatorname{Out}\left(F_{n}\right)$ ?

Acknowledgements. This paper is a branch of the paper [10], and I am extremely grateful to Juan González-Meneses for many helpful discussions.

\section{References}

[1] X. Bressaud, A normal form for braids. J. Knot Theory Ramifications 17 (2008), 697-732. Zbl 1158.20019 MR 2427952

[2] P. Dehornoy, Groupes de Garside. Ann. Sci. École Norm. Sup. (4) 35 (2002), 267-306. Zbl 1017.20031 MR 1914933

[3] P. Dehornoy, I. Dynnikov, D. Rolfsen, and B. Wiest, Ordering braids. Math. Surveys Monogr. 148, Amer. Math. Soc., Providence, RI 2008. Zbl 1163.20024 MR 2463428

[4] I. Dynnikov and B. Wiest, On the complexity of braids. J. Eur. Math. Soc. (JEMS) 9 (2007), 801-840. Zbl 1187.20045 MR 2341833

[5] E. A. El-Rifai and H. R. Morton, Algorithms for positive braids. Quart. J. Math. Oxford (2) 45 (1994), 479-497. Zbl 0839.20051 MR 1315459

[6] R. Fenn, M. T. Greene, D. Rolfsen, C. Rourke, and B. Wiest, Ordering the braid groups. Pacific J. Math. 191 (1999), 49-74. Zbl 1009.20042 MR 1725462

[7] J. Fromentin, Every braid admits a short sigma-definite representative. J. Eur. Math. Soc. (JEMS), to appear; preprint 2008. arXiv:0811.3902

[8] F. A. Garside, The braid group and other groups. Quart. J. Math. Oxford (2) 20 (1969), 235-254. Zbl 0194.03303 MR 0248801

[9] V. Gebhard and J. González-Meneses, Solving the conjugacy problem in Garside groups by cyclic sliding. J. Symbolic Comput. 45 (2010), 629-656. Zbl 05710825 MR 2639308

[10] J. González-Meneses and B. Wiest, Reducible braids and Garside theory. Preprint 2010. arXiv: 1008.0238

[11] H. A. Masur and Y. N. Minsky, Geometry of the complex of curves II: Hierarchical structure. Geom. Funct. Anal. 10 (2000), 902-974. Zbl 0972.32011 MR 1791145

[12] K. Rafi, A combinatorial model for the Teichmüller metric. Geom. Funct. Anal. 17 (2007), 936-959. Zbl 1129.30031 MR 2346280

[13] B. Wiest, An algorithm for the word problem in braid groups. Preprint 2002. arXiv:math/0211169

Received May 5, 2009; revised June 30, 2009

B. Wiest, IRMAR, UMR 6625 du CNRS, Université de Rennes 1, Campus de Beaulieu, 35042 Rennes Cedex, France

E-mail: bertold.wiest@univ-rennes1.fr 